Revista Arbitrada Interdisciplinaria de Ciencias de la Salud. SALUD Y VIDA

Volumen 3. Número 6. Año 3. Julio - Diciembre 2019

Hecho el depósito de Ley: FA2016000010

ISSN: $2610-8038$

FUNDACIÓN KOINONIA (F.K)

Santa Ana de Coro, Venezuela.

Freddy Alberto Ávila Chica; Ruth Verónica Guillen Mendoza; Victor Antonio Avendaño Robles

http://dx.doi.org/10.35381/s.v.v3i6.331

\title{
La anatomía patológica presente y futuro
}

The present and future pathological anatomy

\author{
Freddy Alberto Ávila Chica \\ picosoa@hotmail.com \\ Universidad Laica Eloy Alfaro de Manabí \\ Ecuador \\ https://orcid.org/0000-0003-3000-7281 \\ Ruth Verónica Guillen Mendoza \\ ruth-636153@hotmail.com \\ Universidad Laica Eloy Alfaro de Manabí \\ Ecuador \\ https://orcid.org/0000-0002-2852-4303 \\ Víctor Antonio Avendaño Robles \\ victor avend89@hotmail.com \\ Universidad Laica Eloy Alfaro de Manabí \\ Ecuador \\ https://orcid.org/0000-0002-3690-3151
}

Recibido: 25 de abril de 2019

Aprobado: 26 de mayo de 2019

\section{RESUMEN}

Comenzando con una breve descripción de su historia, se anticipan los desarrollos futuros de la anatomía patológica. Si bien las principales tareas de los institutos de patología en los hospitales residen en la patología clínica, el énfasis de los institutos universitarios está en enseñar y comunicar las bases de la patología general y la anatomía patológica. El progreso requiere un desarrollo más intensivo de investigación experimental y teórica, así como nuevas técnicas morfológicas, aprovechando los recientes avances científicos y técnicos. Una de sus tareas principales debe ser la elaboración de conceptos teóricos cognitivos para desarrollar aún más la comprensión y clasificación de enfermedades y neoplasias, y sus etiologías complejas. El articulo de revisión tiene por objetivo presentar los poses escenarios que enfrentara a anatomía patológicas en su desarrollo y avances como rama de las ciencias de la salud. 
Revista Arbitrada Interdisciplinaria de Ciencias de la Salud. SALUD Y VIDA

Volumen 3. Número 6. Año 3. Julio - Diciembre 2019

Hecho el depósito de Ley: FA2016000010

ISSN: $2610-8038$

FUNDACIÓN KOINONIA (F.K)

Santa Ana de Coro, Venezuela.

Freddy Alberto Ávila Chica; Ruth Verónica Guillen Mendoza; Victor Antonio Avendaño Robles

Descriptores: Anatomía; Avances; Patología; Ciencia; Futuro.

\begin{abstract}
Beginning with a brief description of its history, future developments in pathological anatomy are anticipated. While the main tasks of pathology institutes in hospitals reside in clinical pathology, the emphasis of university institutes is on teaching and communicating the basis of general pathology and pathological anatomy. Progress requires a more intensive development of experimental and theoretical research, as well as new morphological techniques, taking advantage of recent scientific and technical advances. One of its main tasks must be the elaboration of cognitive theoretical concepts to further develop the understanding and classification of diseases and malignancies, and their complex etiologies. The aim of the review article is to present the scenario poses that will face pathological anatomy in its development and advances as a branch of health sciences.
\end{abstract}

Descriptors: Anatomy; Advances; Pathology; Science; Future.

\title{
INTRODUCCIÓN
}

Actuales investigadores han publicado artículos interesantes sobre dos posibles resultados para la patología anatómica en algún momento en el futuro de la anatomía patológica. Un escenario está relacionado con imágenes de diapositivas completas y el otro está relacionado con la microscopía en vivo. La pregunta que surge es: ¿cuál de estos prevalece? En mi opinión, ambos. Habrá menos biopsias "normales" con microscopía in vivo mejorada con menos accesiones de patología, pero las biopsias que se obtienen debido a imágenes in vivo equívocas se examinarán más y se eliminarán de la revisión de patología adicional usando imágenes de diapositivas completas y análisis mejorado que tenemos hoy.

Esto dejará solo una fracción del trabajo actual y terminará en microscopios en los escritorios de los patólogos. Luego hay un tercer resultado: la biopsia líquida. Se eliminarán las biopsias basadas en tejidos que requieren médicos, radiólogos y patólogos. Tendremos que aprender a interpretar los datos morfológicos de los 
Revista Arbitrada Interdisciplinaria de Ciencias de la Salud. SALUD Y VIDA

Volumen 3. Número 6. Año 3. Julio - Diciembre 2019

Hecho el depósito de Ley: FA2016000010

ISSN: $2610-8038$

FUNDACIÓN KOINONIA (F.K)

Santa Ana de Coro, Venezuela.

Freddy Alberto Ávila Chica; Ruth Verónica Guillen Mendoza; Victor Antonio Avendaño Robles

marcadores sustitutos obtenidos in vivo para el diagnóstico, la selección del tratamiento y pronóstico. No seremos patólogos "quirúrgicos", seremos patólogos "líquidos" que competirán para interpretar esta información.

\section{Dos posibles futuros para la patología}

Aquí hay un futuro posible: algún día, los generadores de imágenes de diapositivas completos serán omnipresentes en los laboratorios de patología. Escanearán las diapositivas rápidamente en múltiples planos focales contiguos, conservando así todos los datos morfológicos que existen en forma "analógica". La calidad de la imagen de diapositiva completa será tan buena que los patólogos no tendrán necesidad de revisar las diapositivas originales. Nuestro flujo de trabajo será una proximidad completamente digital y física del laboratorio de histología, el patólogo ya no importará. Los algoritmos de inteligencia artificial interpretarán las diapositivas escaneadas y marcarán los diagnósticos urgentes.

Al principio, el diagnóstico asistido por computadora facilitará el trabajo de los patólogos y podremos hacer más trabajo más rápido y extraer más información de la morfología que antes. Sin embargo, eventualmente los patólogos tendrán menos que hacer a medida que las computadoras se vuelvan más inteligentes y rápidas. En algún momento, la interpretación de diapositivas ya no será realizada por seres humanos, por cuanto la IA puede hacer todo lo que hacen más rápido y son menos propensos a errores. Además, aprenderán a identificar patrones complejos con relevancia diagnóstica y pronostica que están más allá de la percepción humana. Patrones como la distribución de cromatina, la arquitectura de tejido 3D reconstruida y la cuantificación de la irregularidad del contorno nuclear.

Aquí hay otro futuro posible: la microscopía in vivo mejorará hasta el punto en que coincida con la resolución de la histología, excluyendo así la necesidad de una interpretación microscópica ex vivo. Junto con las mejoras en las imágenes metabólicas y el análisis de ADN exosómico, la histología no tendrá nada que aportar 
Revista Arbitrada Interdisciplinaria de Ciencias de la Salud. SALUD Y VIDA

Volumen 3. Número 6. Año 3. Julio - Diciembre 2019

Hecho el depósito de Ley: FA2016000010

ISSN: $2610-8038$

FUNDACIÓN KOINONIA (F.K)

Santa Ana de Coro, Venezuela.

Freddy Alberto Ávila Chica; Ruth Verónica Guillen Mendoza; Victor Antonio Avendaño Robles

más allá de la información que se puede obtener in vivo. La creación de imágenes de diapositivas completas se convertirá en un concepto redundante por cuanto ya no habrá diapositivas para escanear. Los patólogos, radiólogos y médicos que hacen endoscopia competirán por el derecho profesional de interpretar estas imágenes in vivo, lo que hará innecesaria la mayoría de las biopsias.

La mayoría de los departamentos de patología generalmente se organizan en torno a las siguientes líneas de servicio: clínica, anatómica, molecular, investigación, educación e informática. Las líneas fronterizas entre estos diferentes servicios están empezando a difuminarse, mientras se agregan nuevos servicios. Mientras miramos hacia el futuro, los laboratorios de patología y los patólogos necesitarán nuevas competencias en tres áreas:

- Utilizando técnicas moleculares y genómicas apropiadas como parte de su proceso de diagnóstico para mejorar los resultados del paciente.

- Confiar en aplicaciones de software e imágenes electrónicas de diapositivas (también conocidas como patología digital) para realizar diagnósticos y colaborar con sus compañeros.

- Operar en un entorno de equipo altamente subespecializado e integrar varios puntos de datos clínicos para formar un diagnóstico.

Los cambios que llegan a Anatomic Pathology (AP) representan una gran oportunidad para la innovación y el avance tecnológico, pero también conllevan un potencial de interrupción. Adoptar la dinámica del mercado es un paso importante que los laboratorios de AP deberían tomar para aprovechar las oportunidades y minimizar la interrupción. Muchos laboratorios también han encontrado que vale la pena asociarse con un proveedor de software que comprende el espacio altamente especializado de AP. 
Revista Arbitrada Interdisciplinaria de Ciencias de la Salud. SALUD Y VIDA

Volumen 3. Número 6. Año 3. Julio - Diciembre 2019

Hecho el depósito de Ley: FA2016000010

ISSN: $2610-8038$

FUNDACIÓN KOINONIA (F.K)

Santa Ana de Coro, Venezuela.

Freddy Alberto Ávila Chica; Ruth Verónica Guillen Mendoza; Victor Antonio Avendaño Robles

\section{Volver a lo básico}

Por prometedor que sea el futuro, los laboratorios de AP de hoy están preparándose para los próximos cambios en un clima económico difícil. Muchos enfrentan recortes de reembolso, presiones regulatorias y escasez de personal. Por su parte, Jesús Ellin, supervisor de AP y asistente de patología en el Centro Médico Regional de Yuma en Yuma, AZ, frente a la escasez futura, se hizo una simple pregunta, "¿qué puedo hacer hoy para preparar mi laboratorio para el futuro de la patología anatómica?"

La respuesta, afirma, fue notablemente básico: los laboratorios de AP tienen un flujo de trabajo muy manual, de estilo de fabricación. "Debe comenzar a automatizar este flujo de trabajo", dice Ellin. "Debe incorporar códigos de barras de muestras, seguimiento de muestras y procesos ajustados para imprimir diapositivas y casetes. Tienes que construir QA / QC en el LIS para seguir cada paso. Debe convencer a sus patólogos para que usen la computadora en lugar del papel, y debe ayudarlos con la capacitación adecuada y el soporte de TI. Tienes que hacer todo esto antes de comenzar a pensar en la patología digital".

Eso es exactamente lo que hizo Yuma Regional con software e instrumentación de laboratorio de vanguardia. Yuma implementó pasos básicos de automatización del flujo de trabajo. Esto permitió a la organización desplegar con éxito la patología digital aprovechando el código de barras de las muestras y el flujo de trabajo electrónico utilizando el LIS.

El Dr. Mark Tuthill, Jefe de División de Informática de Patología en el Sistema de Salud Henry Ford en Detroit, tiene una opinión similar. "Cuando comencé en HFHS en 2003, no teníamos un verdadero sistema de información de patología anatómica. Hoy confiamos en gran medida en uno para ofrecer operaciones de laboratorio esbeltas y de alta calidad con tiempos de respuesta sorprendentes y un completo etiquetado y seguimiento de activos".

El rendimiento y la seguridad del manejo de muestras se han mejorado enormemente con la institución de los sistemas de seguimiento, señala el Dr. Michael Berman, 
Vicepresidente de Patología en el Centro Médico Regional Jefferson en Crystal City, MO. "Además de ayudar en la auditoría y el seguimiento, su mayor beneficio puede ser la reducción de errores. Utilizan la tecnología de código de barras para garantizar mejor el paso correcto del tejido desde el contenedor de muestras al bloque de parafina, al portaobjetos de vidrio y al escritorio del patólogo".

Con una mayor dependencia de las pruebas moleculares complementarias, la necesidad de manejo de muestras ahora se extiende más allá de las cuatro paredes de la histología. Los procesos manuales en papel se vuelven muy difíciles y propensos a errores, especialmente a medida que aumentan los volúmenes. Esto es cuando cosas como tener a todos los usuarios, incluidos los patólogos, en un sistema central de TI y usar herramientas electrónicas para integrar información y firmar electrónicamente los casos comienzan a ser cada vez más importantes. La tecnología básica del sistema de información es nuevamente muy importante.

Asumamos la patología digital como el estado futuro. Para obtener imágenes de las diapositivas de forma automatizada, debe existir un mecanismo para comunicarse con el sistema de imágenes que se desliza a la imagen y cómo. Esto es muy similar a pedir una mancha, donde el LIS le dice al instrumento qué deslizamiento se mancha con qué tipo de mancha. La identificación única con código de barras se vuelve muy crucial en estos casos.

La patología digital agrega pasos en el flujo de trabajo técnico que pueden tener un efecto negativo en el rendimiento si no se planifica adecuadamente. Por lo tanto, cosas como el tamaño de los lotes, las instrucciones contextuales para los tecnólogos y el seguimiento en tiempo real de las diapositivas se vuelven cruciales para mantener un alto rendimiento.

Otro "problema" con la patología digital, según Ellin, es que puede amplificar los artefactos histológicos. Hoy en día, los patólogos pueden trabajar alrededor de pequeños pliegues y burbujas en un portaobjetos al colocar el portaobjetos bajo un microscopio. Sin embargo, estos artefactos pueden amplificarse y producir 
manifestaciones visuales consecuentes cuando se digitaliza el portaobjetos. Por lo tanto, nuevamente es muy importante contar con una gestión robusta de muestras y procesos de control de calidad para el personal de histología. "EI LIS proporciona el marco para todas estas capacidades", menciona Ellin.

De igual forma, volvamos a la idea de la patología basada en el equipo. Aquí hay una oportunidad real para que los sistemas de TI permitan la colaboración digital. Un caso de médula ósea, por ejemplo, puede incluir, además del diagnóstico morfológico, análisis citogenéticos adicionales, análisis moleculares, citometría de flujo e inmunohistoquímica. Estos diferentes pasos son realizados por diferentes miembros del equipo. Eso requiere coordinación y comunicación de tareas e integración de resultados para un estudio de caso exitoso.

Los procesos manuales y en papel se descomponen rápidamente en tales escenarios, y se requiere el uso de tecnología de la información para ser cada vez más sofisticado. Tiene sentido que la evolución de la patología indudablemente conduzca a un producto final mejorado y más adaptable, "el santo grial del patólogo", el informe final. El Dr. Berman cree que esta nueva especie de informes está en marcha.

\section{El futuro de la patología.}

El cambio rápido se ha convertido en una característica definitoria de la patología, pero ¿puede este cambio impulsar una nueva generación de software de laboratorio para configurar el papel del laboratorio clínico del futuro? El cambio rápido se ha convertido en una característica definitoria de la patología, pero ¿puede este cambio impulsar una nueva generación de software de laboratorio para configurar el papel del laboratorio clínico del futuro?

No será una sorpresa para aquellos en el campo del laboratorio clínico y la patología que el mercado esté experimentando un cambio rápido. En las últimas décadas, las expectativas de salud han aumentado a nivel mundial, con todos los estados miembros de la Organización Mundial de la Salud comprometidos a trabajar para lograr una 
cobertura de salud universal en todo el mundo. La escala adecuada de los servicios de patología es clave para este crecimiento, ya que la patología está involucrada en el $70 \%$ de todos los diagnósticos de atención médica. Si el mercado de patología sigue la tasa de crecimiento anual compuesta del 6,8\% entre 2014 y 2020, según lo previsto en un nuevo estudio realizado por los analistas de mercado Grand View Research1, se espera que el mercado mundial de laboratorios clínicos alcance los 149 mil millones de dólares en 2020.

Junto con el auge de la atención médica universal, otros factores están impulsando el cambio en el mercado de patología. Estos incluyen un envejecimiento de la población y la creciente prevalencia de afecciones crónicas como la obesidad y la diabetes. También estamos viendo un aumento de los nuevos métodos de prueba para apoyar iniciativas como la medicina personalizada, también conocida como medicina genómica, y las pruebas de punto de atención. Al igual que aumenta la demanda de servicios de laboratorio, existe una presión a la baja para utilizar menos recursos. La consolidación de la industria es tan frecuente en los laboratorios como en otras áreas de la atención médica, y se espera que el aumento de las economías de escala ofrezca más resultados de pruebas a un costo menor.

Lo bueno es la abundancia de información valiosa que fluye del ciclo de vida de las pruebas médicas. La informática de la salud ahora puede capturar esta información en cada etapa de la continuidad de la atención médica para ofrecer comentarios en tiempo real para mejorar el proceso y la gestión general. Estas tendencias presentan un desafío formidable para muchos de los sistemas de gestión de información de laboratorio (LIMS) existentes. La mayoría fueron diseñados simplemente para automatizar la solicitud, la prueba y el informe en un proceso lineal vinculado a ubicaciones fijas, procesos y flujos de trabajo. Sin nuevos tipos de LIMS, los futuros laboratorios no podrán adaptarse y responder al cambio que se les exige.

En cambio, los servicios de patología necesitarán sistemas de información moderna y ágiles que brinden la funcionalidad para capturar, compartir, analizar y actuar sobre 
Revista Arbitrada Interdisciplinaria de Ciencias de la Salud. SALUD Y VIDA

Volumen 3. Número 6. Año 3. Julio - Diciembre 2019

Hecho el depósito de Ley: FA2016000010

ISSN: $2610-8038$

FUNDACIÓN KOINONIA (F.K)

Santa Ana de Coro, Venezuela.

Freddy Alberto Ávila Chica; Ruth Verónica Guillen Mendoza; Victor Antonio Avendaño Robles

grandes cantidades de datos detallados. Estos nuevos sistemas deberán funcionar de formas mucho más diversas y flexibles, moldeadas por el servicio de patología necesario en lugar de tratar de moldear el servicio al sistema. Entonces, ¿qué deben hacer los servicios de patología para adaptarse a estos desafíos en el clima donde es probable que se acelere el ritmo del cambio?

\section{Medicina personalizada}

La medicina personalizada, también conocida como medicina genómica o medicina de precisión, es un campo en rápido crecimiento. A través de los avances en genética y genómica, ahora es posible identificar la predisposición a la enfermedad o realizar diagnósticos a nivel genético de ciertos tipos de cáncer, como el gen BRAC. Al mismo tiempo, los precios de este tipo de pruebas están disminuyendo; la ciencia en sí misma sigue siendo costosa, pero ahora es posible mapear un genoma humano por tan solo $\$$ 1,000 .

Los avances continuarán y los precios seguirán cayendo, de modo que la entrega de medicamentos personalizados se convertirá en una expectativa mucho más común para los médicos. Esto, a su vez, tendrá un impacto significativo en los servicios de patología tradicionales y cambiará la forma en que los laboratorios realizan pruebas. La ciencia de la genética obligará a las unidades de patología y los servicios de laboratorio a instalar instalaciones más avanzadas, proporcionar capacitación en nuevos conjuntos de habilidades y reclutar personal que tenga el conocimiento y la experiencia para interpretar y diagnosticar en función de nuevos resultados e información.

La medicina personalizada transformará por completo el alcance del servicio de patología y su beneficio potencial para los servicios de salud. Tomemos, por ejemplo, un paciente que ha experimentado un derrame cerebral. Con una inversión de $\$ 1,000$ para comprender el genoma del paciente, los patólogos clínicos pueden identificar rápida y fácilmente el mejor anticoagulante para el cuidado posterior. Esto podría reducir el tiempo que el paciente pasa en el hospital y mejorar el período de 
Revista Arbitrada Interdisciplinaria de Ciencias de la Salud. SALUD Y VIDA

Volumen 3. Número 6. Año 3. Julio - Diciembre 2019

Hecho el depósito de Ley: FA2016000010

ISSN: $2610-8038$

FUNDACIÓN KOINONIA (F.K)

Santa Ana de Coro, Venezuela.

Freddy Alberto Ávila Chica; Ruth Verónica Guillen Mendoza; Victor Antonio Avendaño Robles

recuperación, al tiempo que ayuda a identificar el mejor protocolo de rehabilitación en la comunidad.

El resultado no es solo un mejor resultado y experiencia para el paciente, sino un ahorro de costos muy necesario. Claramente, podría haber un gran beneficio para el NHS. Sin embargo, la inversión para crear esta capacidad personifica la necesidad de una visión a largo plazo. Los costos iniciales para crear laboratorios con esta capacidad son considerables, y estos no son los únicos desafíos que la medicina personalizada crea para los servicios de patología del NHS.

Los laboratorios que utilizan la tecnología de secuenciación genética también deben considerar una mayor demanda de seguridad de estos datos altamente personales del paciente. Se necesitan protocolos estrictos para manejar la custodia de las muestras. Por lo tanto, los laboratorios enfrentarán controles más rígidos, más auditorías y más preguntas sobre el uso y manejo de las muestras. Además, los servicios de medicina personalizada impulsarán la demanda de científicos clínicos con capacitación especializada que actualmente no es satisfecha por el ecosistema educativo, exacerbando los problemas de reclutamiento y retención de personal.

\section{Apoyando a una población que envejece}

A medida que las personas vivan más tiempo y sobrevivan enfermedades como el cáncer, necesitarán apoyo para manejar la vida con condiciones crónicas. Muchas de las personas mayores de la actualidad gozan de buena salud después de haberse sometido a un procedimiento, pero otras son frágiles y no envejecen con buena salud. Esto genera una serie de presiones en el servicio general del NHS, pero para los servicios de patología significará más pruebas y, fundamentalmente, más pruebas de rutina, lo que constituirá una proporción cada vez mayor de la carga de trabajo.

Es probable que el aumento de las pruebas de rutina conduzca a una automatización aún mayor. El impacto para los laboratorios será que el "centro" en el modelo de centro y radio se parecerá más a las fábricas, con estos laboratorios manejando grandes 
volúmenes de pruebas esenciales pero rutinarias, como recuentos sanguíneos y monitoreo del colesterol. En regiones con una población más grande de ciudadanos mayores, esperaríamos ver más laboratorios "hablados" conectados que ayuden a gestionar la demanda en múltiples entornos de atención primaria y secundaria. Si se implementa con éxito, un modelo de concentrador y radio con automatización y transferencia electrónica de datos es más rápido y más seguro.

Los resultados en este modelo deben entregarse rápidamente a los equipos de atención, entregando automáticamente los resultados directamente en el registro del paciente. Los clientes (médicos, hospitales, médicos de cabecera, etc.) esperarán acceso instantáneo a los resultados a través de sus navegadores de Internet, utilizando los últimos dispositivos móviles, sujetos a los protocolos de seguridad apropiados. Por lo tanto, serán necesarios nuevos sistemas y arquitectura para la gestión de la información para gestionar este acceso fácil pero seguro.

\section{El punto de atención}

Es probable que veamos más pruebas de punto de atención (PoCT), no solo en las prácticas de GP y en el hogar, sino siempre que sea óptimo y asequible en el protocolo de atención. Las pruebas de rutina ahora se pueden realizar fácilmente con PoCT, y qué tan bien los laboratorios los acomoden con el flujo de trabajo requerido y los cambios de gobernanza serán un marcador clave de cuán bien podrán adaptarse y cambiar con los tiempos.

Existe una gran demanda de PoCT, no solo de los profesionales de la salud sino también del público. Quizás lo más visible es la explosión de aplicaciones de salud para teléfonos inteligentes. Todos los días se anuncian dispositivos telefónicos disponibles que realizan pruebas de rutina, como pruebas de glucosa y monitoreo de la frecuencia cardíaca. Esto ya está transformando la forma en que las personas con afecciones crónicas manejan su atención de una manera no prevista hace solo una década. Los servicios de patología no pueden ignorar la demanda del consumidor, y no solo 
necesitan aceptar sino también administrar los muy diferentes dominios de datos de dispositivos clínicos de bienestar, terapéuticos y validados y calibrados.

Esencialmente, aunque el papel de los laboratorios en la entrega de resultados instantáneos evolucionará, su papel esencial para defender y hacer cumplir la gobernanza de los datos clínicos de diagnóstico y su uso debe permanecer. Esta es la única forma predecible y sostenible de garantizar que la información recopilada de PoCT se utilice para proporcionar resultados seguros y positivos para el paciente.

Para aprovechar los beneficios de las nuevas opciones de pruebas y mitigar los desafíos de nuestra población que envejece, será de vital importancia configurar todo el modelo de atención en torno a los pacientes, colocando a los pacientes en el centro de atención. Para hacer esto de manera efectiva, los laboratorios necesitarán adoptar sistemas de información y gestión empresarial que respalden inherentemente la atención conectada con un registro de pacientes de patología contigua (PPR).

Esto formará parte de la historia clínica electrónica del paciente si el laboratorio forma parte de un grupo de atención médica, y se integra con las historias clínicas electrónicas compartidas regionales o nacionales. EI PPR incluiría todos los datos de prueba relevantes para los pacientes, incluso los resultados de las pruebas en el punto de atención. En última instancia, se requiere un nuevo enfoque de la informática de laboratorio para respaldar este trabajo, que es realmente adecuado para su propósito y refleja las demandas del nuevo entorno.

Las asociaciones y las redes de patología que unen tanto a los laboratorios del sector público como al privado están surgiendo en el modelo de prestación de servicios de salud. Dichas asociaciones presentan una clara oportunidad para apoyar las necesidades del servicio de salud del futuro. De hecho, las redes de laboratorios ofrecen una de las mejores oportunidades para la colaboración pública y privada al ayudar a hacer un mejor uso de los recursos en una región. Aportan una serie de beneficios en torno a la eficiencia de ahorro de costos y la optimización eficaz de los recursos. 
Una red permite la intercambiabilidad del personal, lo que permite que las personas se vuelvan a implementar donde sea necesario durante los picos en el flujo de trabajo. InterSystems cree que la formación de redes será clave para futuras inversiones en patología, tanto dentro del NHS como externamente. De la misma manera que los sistemas de gestión de la información serán cruciales para aprovechar los beneficios de la medicina personalizada, también serán esenciales para permitir la adopción exitosa de redes de laboratorio. Modelos como este requieren claramente un nuevo enfoque que permita compartir e integrar información y flujos de trabajo en múltiples entornos de atención.

Quizás el mayor desafío para los servicios de patología no sea que la demanda esté aumentando o que el descubrimiento científico esté abriendo una nueva oportunidad. El cambio fundamental requerido es que los laboratorios, al tiempo que garantizan la calidad de los resultados de los pacientes dentro de sus presupuestos operativos, deben adoptar un enfoque centrado en el negocio para la prestación de atención médica.

La patología ha sido históricamente un servicio impredecible de demanda y oferta. Con un enfoque comercial, los laboratorios implementarán sus protocolos de flujo de trabajo (o procedimientos operativos estándar) y le costarán cada procedimiento. Los directores clínicos y gerentes comerciales llevarán a cabo un negocio afinado e impulsado por la información con informática que les brinde visibilidad, control y responsabilidad sobre los procesos comerciales.

El rendimiento del laboratorio y los tiempos de respuesta serán monitoreados constantemente. El software identificará los cuellos de botella del flujo de trabajo sin la necesidad de análisis personalizados. Cada laboratorio o grupo podrá configurar fácilmente los indicadores clave de rendimiento relevantes y relacionarlos con los acuerdos de nivel de servicio y monitorearlos de cerca con paneles y alertas. Cuando los sistemas de laboratorio pueden capturar y acceder a información relevante a lo largo de cada paso del proceso de prueba, también ofrecerán una capacidad sin 
Revista Arbitrada Interdisciplinaria de Ciencias de la Salud. SALUD Y VIDA

Volumen 3. Número 6. Año 3. Julio - Diciembre 2019

Hecho el depósito de Ley: FA2016000010

ISSN: $2610-8038$

FUNDACIÓN KOINONIA (F.K)

Santa Ana de Coro, Venezuela.

Freddy Alberto Ávila Chica; Ruth Verónica Guillen Mendoza; Victor Antonio Avendaño Robles

precedentes para predecir y administrar cargas de trabajo variables. Por ejemplo, cuando un clínico completa una orden de prueba de patología, incluso antes de que la muestra haya sido tomada del paciente, la solicitud se incorporará al flujo de trabajo del laboratorio para mejorar la previsibilidad de las cargas de trabajo.

La medicina de laboratorio hoy es una de las mayores fuentes de información clínica. Es fundamental que los profesionales de laboratorio tengan la capacidad de analizar, dividir y cortar la información para optimizar su papel en el modelo de prestación de atención médica de hoy, y transformarse para liderar el servicio de patología del futuro. Los laboratorios y el papel vital que desempeñan en la ciencia de la medicina están ahora en el punto de inflexión.

Se encuentran entre las pruebas tradicionales basadas en el volumen y el nuevo mundo de pruebas distribuidas y resultados centrados en el paciente para la atención médica personalizada. Las oportunidades para los servicios de patología quizás nunca hayan sido mayores: para la colaboración en la atención, la adopción de pruebas en el punto de atención e impulsar la evolución de la medicina con las pruebas genómicas.

Sin embargo, deben sobrevivir a las demandas de apoyar a una población en constante cambio y presiones económicas pragmáticas. Esto requiere una nueva generación de sistemas informáticos de salud que satisfagan las necesidades de un entorno patológico moderno. Armados con tales sistemas, los profesionales de laboratorio y los servicios de patología que brindan dejarán de ser víctimas de la interrupción del mercado; en cambio, se convertirán en los facilitadores clave de los cambios que anunciarán una nueva era de la medicina. Algunos autores señalan las siguientes direcciones futuras a la prestación del servicio de patología:

1. Agilización de los flujos de trabajo para aumentar la eficiencia operativa o desarrollar un servicio central de laboratorio para mejorar la capacidad de satisfacer la demanda futura

Los avances recientes en la automatización ahora permiten la integración del manejo pre-analítico y post-analítico de muestras y el procesamiento analítico para pruebas 
de alto volumen para una serie de áreas de patología, como Patología Química, Hematología, Coagulación, Microbiología, Serología, Endocrinología e Inmunología. Estas pruebas formarán el perfil de prueba futuro de Pruebas de "laboratorio central". Además, una serie de pruebas manuales y semiautomáticas en muchas áreas de laboratorio también serán automatizadas.

Aumentar la capacidad de plataformas tecnológicas preexistentes de especialidad cruzada: Los avances tecnológicos han proporcionado equipos y técnicas que se pueden utilizar para las pruebas de patología en las disciplinas de patología tradicionales. Como resultado, hay una serie de plataformas tecnológicas altamente especializadas (como citometría de flujo y cromatografía, espectrometría de masas y secuenciación) con capacidad no utilizada.

El uso de la robótica y la automatización en el procesamiento de pruebas: Se podrá investigar sobre los avances en robótica y automatización pudiendo mejorar la prestación de servicios de diagnóstico al eliminar los pasos de procesamiento manual y reducir la variación técnica en las pruebas. Es probable que esta tecnología también tenga aplicaciones que pueden ser utilizadas por más de un flujo clínico.

2. Crear estándares estatales para mejorar nuestra agilidad Hasta hace poco, los procesos de laboratorio y la interpretación de los resultados para muchas pruebas de patología se han desarrollado de forma aislada de pruebas similares en otros laboratorios. En el futuro se podrá facilitar un proceso para la estandarización estatal de los nombres de prueba, procesos de prueba, intervalos de prueba y armonización de equipos de prueba. Esto reducirá la duplicación y mejorará la transferibilidad de los servicios de patología.

3. Adopción de nuevas tecnologías e innovaciones

La implementación de nuevas tecnologías debe estar en marcha dentro de las operaciones clínicas. El uso de la tecnología de imagen digital garantizará el acceso a expertos en las subespecialidades dentro de Microbiología, Anatomía Patológica y 
Hematología. Los patólogos podrán proporcionar rápidamente asesoramiento y consulta a los médicos en los centros de salud.

El uso creciente de técnicas moleculares junto con el uso de espectrometría de masas para biomarcadores proteómicos y metabólicos en las pruebas de diagnóstico de rutina aprovechará la experiencia en patología en la atención médica. En particular, el mayor impacto será en el apoyo a protocolos de atención y tratamiento preventivos personalizados en servicios de oncología y enfermedades crónicas.

4. Aprovechamiento de la experiencia y habilidades

La fuerza laboral tiene un conocimiento y experiencia significativos en patología. Se debe utilizar las innovaciones tecnológicas para obtener la máxima ventaja para facilitar el acceso a expertos reconocidos en las subespecialidades específicas.

5. La investigación en patología para informar mejores resultados para los pacientes. La investigación y la innovación son un área central así como una cultura de investigación e innovación se debe integrar en las actividades de entes gubernamentales y centros de salud. Como resultado, estos se posicionarán a la vanguardia del desarrollo de nuevas pruebas y tecnologías emergentes al apoyar activamente la traducción de los resultados de la investigación a la práctica clínica.

\section{REFERENCIAS CONSULTADAS}

1. Ding, Y., Wang, H., Shen, H., Li, Z., Geng, J., Han, H., et al. (2003) La patología clínica del síndrome respiratorio agudo severo (SRAS): un informe de China. J. Pathol.

2. Duerden, B. y Collee, J. (2006) Microbiology and the Pathological Society. En el centenario de la Sociedad Patológica de Gran Bretaña e Irlanda (eds P. A. Hall y N. A. Wright).

3. Hilton, D. A., Ghani, A. C., Conyers, L., Edwards, P., McCardle, L., Ritchie, D., et al. (2004) Prevalencia de la acumulación de proteína priónica linforreticular en muestras de tejido del Reino Unido. J. Pathol.

4. Toner, P. y Herrington, C. S. (2002) Un nuevo sistema editorial para la revista de patología. J. Pathol. 
Revista Arbitrada Interdisciplinaria de Ciencias de la Salud. SALUD Y VIDA

Volumen 3. Número 6. Año 3. Julio - Diciembre 2019

Hecho el depósito de Ley: FA2016000010

ISSN: $2610-8038$

FUNDACIÓN KOINONIA (F.K).

Santa Ana de Coro, Venezuela.

Freddy Alberto Ávila Chica; Ruth Verónica Guillen Mendoza; Victor Antonio Avendaño Robles

5. Toner, P. G. y Reece, D. (2000a) Into 2000: Revista de patología en la web. J. Pathol.

6. Virchow, R. (1893) Transformación y descenso. J. Pathol. Bacteriol

7. Walker, F. (2006) personas 1980's: Eventos y reuniones. En Centenario de la sociedad de patología de Gran Bretaña e Irlanda. (Eds P. A. Hall y N. A. Wright). Wiley: Chichester.

\section{REFERENCES CONSULTED}

1. Ding, Y., Wang, H., Shen, H., Li, Z., Geng, J., Han, H., et al. (2003) The clinical pathology of severe acute respiratory syndrome (SARS): a report from China. J. Pathol.

2. Duerden, B. and Collee, J. (2006) Microbiology and the Pathological Society. On the centenary of the Pathological Society of Great Britain and Ireland (eds P. A. Hall and N. A. Wright).

3. Hilton, D. A., Ghani, A. C., Conyers, L., Edwards, P., McCardle, L., Ritchie, D., et al. (2004) Prevalence of the accumulation of lymphoreticular prion protein in tissue samples from the United Kingdom. J. Pathol.

4. Toner, P. and Herrington, C. S. (2002) A new editorial system for the pathology journal. J. Pathol.

5. Toner, P. G. and Reece, D. (2000a) Into 2000: Journal of pathology on the web. J. Pathol.

6. Virchow, R. (1893) Transformation and descent. J. Pathol. Bacteriol

7. Walker, F. (2006) 1980's people: Events and meetings. In Centenary of the pathology society of Great Britain and Ireland. (Eds P. A. Hall and N. A. Wright). Wiley: Chichester. 